\title{
Facial Tightening Following a Single Acoustic Wave Treatment Using Focused and Radial Acoustic Waves
}

\author{
Kuriko Kimura1 $^{*}$, Yohei Tanaka1,2 \\ ${ }^{1}$ Health \& Beauty Clinic Le Coquelicot, Tokyo, Japan \\ ${ }^{2}$ Clinica Tanaka Plastic, Reconstructive Surgery and Anti-Aging Center, Matsumoto, Japan \\ Email: *kuriko_k@coquelicot.co.jp
}

How to cite this paper: Kimura, K. and Tanaka, Y. (2019) Facial Tightening Following a Single Acoustic Wave Treatment Using Focused and Radial Acoustic Waves. Journal of Cosmetics, Dermatological Sciences and Applications, 9, 321-328. https://doi.org/10.4236/jcdsa.2019.94029

Received: November 4, 2019

Accepted: December 3, 2019

Published: December 6, 2019

Copyright (@) 2019 by author(s) and Scientific Research Publishing Inc. This work is licensed under the Creative Commons Attribution International License (CC BY 4.0).

http://creativecommons.org/licenses/by/4.0/

\begin{abstract}
Background: Acoustic wave has been used for many medical purposes, and although it is widely applied in cosmetic procedures, it has not been conducted for facial rejuvenation and tightening. Acoustic wave has been proven to penetrate deeper than subcutaneous tissue, independent of skin color, and to be beneficial for skin tightening and body contouring. Objective: To evaluate the effectiveness of a single acoustic wave treatment session using unique focused and radial acoustic waves for facial tightening. Participants: Sixty Asian patients were treated between the years 2016 and 2018. The single acoustic wave treatment session consists of focused long planar acoustic pulses thought to have an effect on cells and their metabolism resulting in an increased release of fat, and radial short acoustic pulses thought to have an effect on tissues activating blood and lymphatic flow. Patients did not undergo any form of medical aesthetic treatment during the study. Measurements: Digital photographs and three-dimensional imaging were used to evaluate the results. Results: Objective assessments with digital photographs and superimposed three-dimensional color images showed preferable facial tightening effects and marked volumetric reduction on the lower one-third of the face. These results were obtained without the edematous reactions observed in laser and radiofrequency therapies, and were sustained up to 12 months. All of the patients reported satisfaction with the results and convenience of the procedure. Complications were minor and transitory, consisting of a slight tingly sensation to the teeth during the treatment, and mild erythema resolving within 5 hours. Side effects were not observed. Conclusions: The advantages of the single acoustic wave treatment are the high potency of facial tightening effects, reduction of discomfort and side effects. Taken together, these characteristics facilitate the ability to give repeated treatments and provide an alternative or adjunct treatment for patients with improved results.
\end{abstract}




\section{Keywords}

Acoustic Wave Treatment, Facial Tightening, Focused Acoustic Waves, Radial Acoustic Waves, Volumetric Reduction

\section{Introduction}

Demand for a non-invasive and long-lasting treatment to reduce undesirable fat tissue and to induce skin tightening has grown dramatically over the past few decades as new aesthetic technologies have been introduced into practice [1] [2]. Regardless of age and skin type, clinical signs of skin ageing and skin laxity are major common complaints among patients seeking rejuvenation and cosmetic procedures [1] [2].

Recently, acoustic wave technology has been introduced in the cosmetic and aesthetic field. Acoustic wave treatment has been initially used to treat kidney stones [3] [4] and injuries in the musculoskeletal system and soft tissue [5] [6] [7]. The positive effects observed on skin and subcutaneous tissue after acoustic wave treatments led to be applied for cosmetic and aesthetic purposes [8].

Acoustic wave is a non-invasive technique based on the propagation of mechanical waves into the tissues [8]. It stimulates local blood circulation, cell metabolism, self-regenerating processes [9] [10], and cell proliferation of collagen and elastin fibres [9] [10] [11], and lipolysis [11]. It is applied for the treatment of localized fat deposits in which high-intensity ultrasound waves disrupt the cellular membrane of adipocytes by causing shear stress and inducing lipolysis [12]. Improvement in the appearance of both cellulite and skin firmness after acoustic wave treatments was reported [8] [12] [13].

Although acoustic wave has been widely used for many medical purposes and reported extensively by previous researchers, cosmetic effects for facial rejuvenation and tightening have not been reported.

A significant limitation in studies designed to measure facial tightening effects is the absence of an agreed standard for precisely capturing the levels of observed effects. Two-dimensional (2-D) imaging has been widely deployed, but may not represent an unquestionably accurate objective representation. In the author's prior studies, this limitation was overcome by the addition of a superimposed three-dimensional (3-D) color schematic scan to increase data precison, as well as demonstrate results not visible in 2-D imagery [1] [2] [14] [15].

In this study, the single acoustic wave treatment session using unique focused and radial acoustic waves was performed, and provided definitive clinical evidence of facial tightening objectively.

\section{Methods}

\subsection{Asian Patients}

Sixty Asian patients (53 females and 7 males) aged 23 - 82 years (mean age, 49.55 
\pm 10.07 years) with Fitzpatrick skin type III-IV were enrolled in this study. All of the patients had visited the Health \& Beauty Clinic Le Coquelicot to reduce undesirable fat tissue and to seek facial tightening, and were treated between the years 2016 and 2018. None of the patients had a history of any type of skin disease or cosmetic procedure that affected the treatment areas within 3 years of this study. Patients did not use any specific skin care products and had no specific diet. Additionally, they were instructed to continue with their normal diet throughout the study. As this study involved a retrospective review of patients previously treated, the approval of an ethics committee was not required.

Patients who exhibited weight loss during the study period were excluded from volumetric measurement analyses because changes in diet and/or exercise may affect volumetric changes. All patients signed an informed consent document of participation following an explanation of the study design and execution, and agreed publication of results and images.

\subsection{Acoustic Wave Treatment}

Acoustic wave device used in the study was a Cellactor SC1 (Storz Medical AG, Tägerwilen, Switzerland), which is capable of delivering 2 sources of acoustic waves through 2 different applicators.

The planar handpiece generates long deeper acoustic pulses electromechanically. This is thought to have an effect on cells and their metabolism, ultimately resulting in an increased release of fat [13]. The maximum energy level range was from 0.45 to $0.88 \mathrm{~mJ} / \mathrm{mm}^{2}$ with a pulse frequency of $3-5 \mathrm{~Hz}$.

The radial handpiece generates short, radial acoustic pulses pneumatically, activating blood, and lymphatic flow at the skin surface [13]. The maximum energy range was from 4 bar with a frequency of $21 \mathrm{~Hz}$.

The treatment consisted of 3000 pulses delivered by the planar handpiece followed by 4000 pulses delivered by the radial handpiece.

Ultrasound coupling gel was applied during each treatment to ensure efficient transmission of energy and smooth movement of the applicators on the skin. No oral analgesics were administered before, during, or after the treatment. In addition, no skin cooling was required. All the treatment sessions were applied by the same trained professional.

\subsection{Objective Assessments}

Digital photographs and 3-D imaging were used as objective assessments and acquired with a Canfield Scientific Vectra Handy camera and software (Canfield Scientific Inc., Fairfield, NJ). This system is designed to accurately capture the surface shape and also 2-D color information of the human body. The superimposed 3-D color schematic representations were used to indicate the volumetric changes between pre-treatment and post-treatment, and shows the varying degrees of tightening in colors that range from yellow to red. Green areas indicated no changes to the face. 3 -D volumetric assessments were performed up to 12 months after the treatment. 


\section{Results}

Objective assessments with digital photographs and superimposed 3-D color images showed marked volumetric reduction for all patients. Representative 2-D color images and superimposed 3-D color images are shown in Figures 1-3. Preferable facial tightening effects and volumetric reduction on the lower one-third of the face were observed through superimposed 3-D color schematic representations. These results were obtained without the edematous reactions observed in laser and RF therapies, and were sustained up to 12 months. All of the patients reported satisfaction with the results and convenience of the procedure.

Complications were minor and transitory, consisting of a slight tingly sensation to the teeth during the treatment in 40 patients, and mild erythema resolving within 5 hours in 2 patients. The slight tingly sensation to the teeth was $\mathrm{mi}$ tigated with cotton filling and resolved immediately after the treatment. Most of the patients did not report feeling pain during the treatment, even though it was performed without anesthesia and contact cooling. Side effects, such as epidermal burns, adipose tissue atrophy, and contraction, were not observed throughout the study.

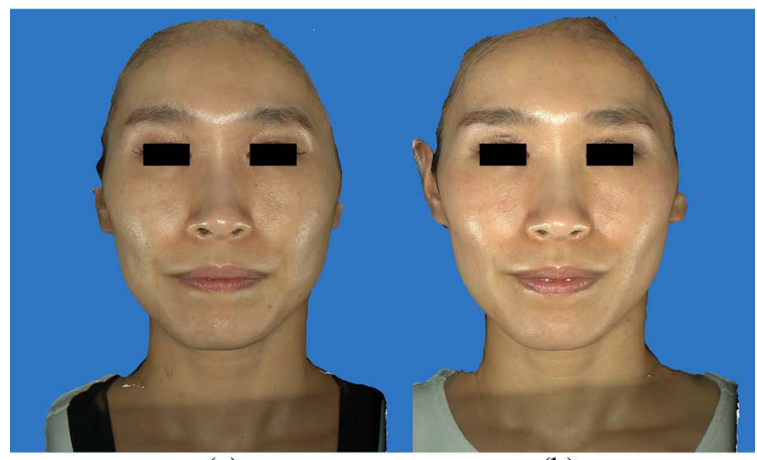

(a)

(b)

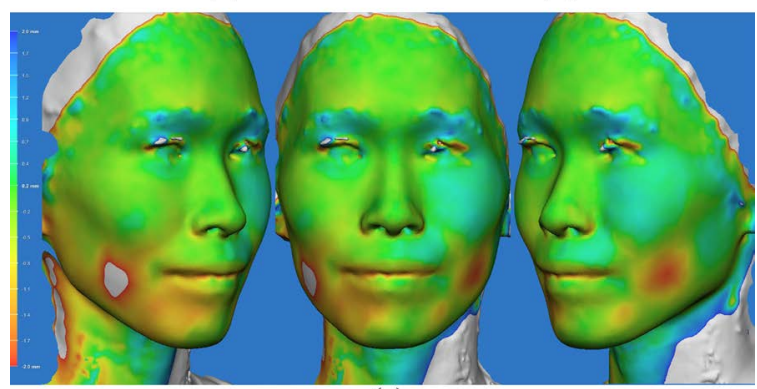

(c)

Figure 1. Representative photographs of the single acoustic wave treatment, before (a) and after treatment (b). A 36-year-old Japanese female. Marked improvement of skin laxity was observed in 2-D color digital photographs compared to the pre-treatment ((a), (b)). Volumetric reduction in superimposed 3-D volumetric assessment was observed 1 month after the treatment compared to the pre-treatment (c). The varying degrees of tightening achieved are shown in colors yellow to red (red, $-2.0 \mathrm{~mm}$ change). Green areas indicated no changes to the face. Tightening effects on the lower one-third of the face that were induced by the treatment lasted for 12 months. 


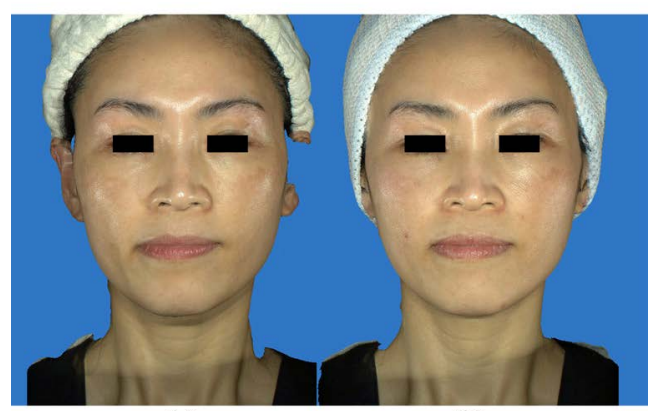

(a)

(b)

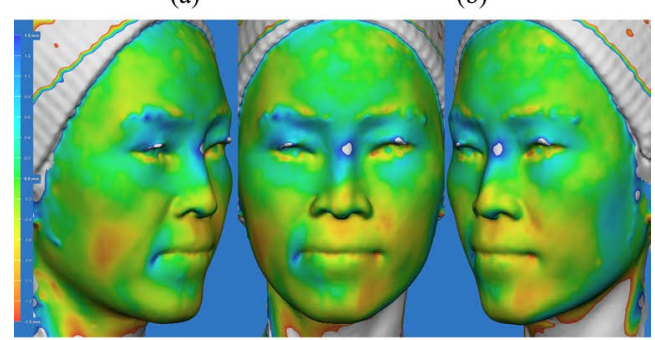

(c)

Figure 2. Representative photographs of the single acoustic wave treatment, before (a) and after treatment (b). A 41-year-old Vietnamese female. Marked improvement of skin laxity was observed in 2-D color digital photographs compared to the pre-treatment ((a), (b)). Volumetric reduction in superimposed 3-D volumetric assessment was observed 1 month after the treatment compared to the pre-treatment (c). Green areas indicated no changes to the face, and gray areas indicate changes over $-1.5 \mathrm{~mm}$. Tightening effects on the lower one-third of the face that were induced by the treatment lasted for 12 months.

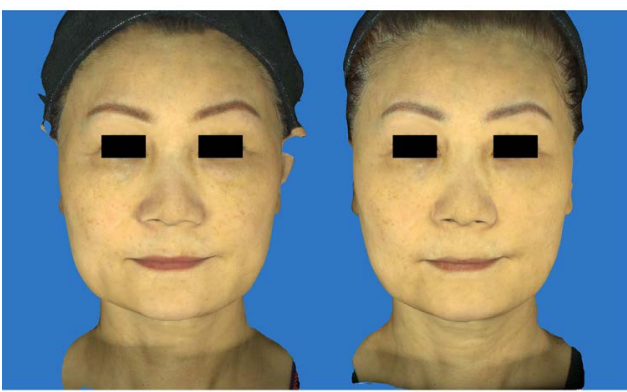

(a)

(b)

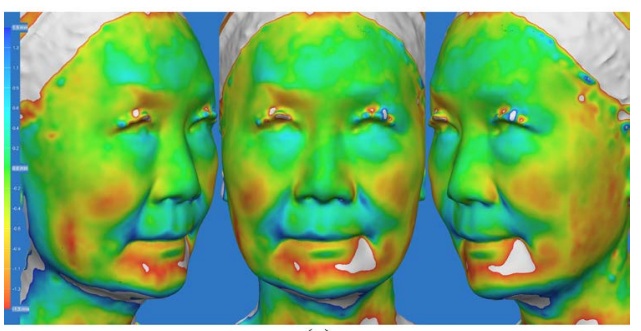

(c)

Figure 3. Representative photographs of the single acoustic wave treatment, before (a) and after treatment (b). A 51-year-old Korean female. Marked improvement of skin laxity was observed in 2-D color digital photographs compared to the pre-treatment ((a), (b)). Volumetric reduction in superimposed 3-D volumetric assessment was observed 1 month after the treatment compared to the pre-treatment (c). Green areas indicated no changes to the face, and gray areas indicate changes over $-1.5 \mathrm{~mm}$. Tightening effects on the lower one-third of the face that were induced by the treatment lasted for 12 months. 


\section{Discussion}

Treatments for facial contours and skin laxity are one of the most common requests among cosmetic patients. Although invasive procedures, such as liposuction or surgeries, are effective to improve contours and to reduce undesirable fat tissue, the downtime and scar formation are not well accepted [15]. Ablative procedures, such as laser resurfacing, are effective in skin tightening, however the downtime and potential adverse effects, such as hyper- and hypo-pigmentation are not well accepted [15]. Noninvasive facial tightening procedures can be particularly applicable to Asians with colored skin, because these procedures are skin-type independent [16].

Acoustic wave therapy has been used in urology for the disintegration of kidney stones and in the treatment of orthopedic conditions for over 30 years [11]. More recently, acoustic wave therapy has been found to strengthen connective tissue and improve skin elasticity. Studies examining its effects on the reduction of localized adipocytes and cellulite have shown promising results. Acoustic wave therapy emits short radial pulses that stimulate angiogenesis, and long planar pulses that result in cell membrane destruction by causing shear stress [13].

The authors evaluated the efficacy of the single acoustic wave treatment objectively, and found that the treatment could provide satisfactory results without side effects.

Nonsurgical volumetric reduction and skin tightening are usually achieved by radiofrequency (RF), near-infrared (NIR), or other thermal approaches. The author has previously reported that these treatments could provide impressive results shown through 3-D volumetric assessment in prior studies [1] [2] [14]. However, these treatments are always accompanied by thermal damage to the dermis, and edematous reactions that can last up to 2 months after the treatment [2] [15].

Interestingly, only single acoustic wave treatment provided the preferable facial tightening effects and improvement of various skin conditions without thermal damage and edematous reactions reported in our prior RF or NIR studies [2] [15].

Although the volumetric assessment was performed up to12 months after the treatment, marked volumetric reduction and facial tightening were observed compared to the pre-treatment in all of the patients. Since the effects of the single acoustic wave treatment are clinically observed for at least 12 months after the treatment, further studies of volumetric assessments with a longer follow-up time are needed.

Furthermore, all patients were satisfied with the improvements in skin laxity, did not report feeling pain during the treatment, even though it was performed without anaesthesia and contact cooling.

Side effects, such as epidermal burns, adipose tissue atrophy, and contracture, were not observed throughout the study. Further studies are necessary to deter- 
mine if a higher intensity, increased frequency of treatments, or longer periods of treatment may be even more effective.

It should be noted that this was a preliminary study based on a fairly small number of patients. Moreover, we cannot exclude the possibility that lifestyle habits, such as food, alcohol and salt intake may affect the changes observed in the current study. Therefore further studies in this area are warranted in a larger number of patients and with longer post-treatment periods to evaluate variations in treatment parameters and correlations with patients' environmental factors.

\section{Limitations}

Lack of a control group and lack of a comparison between dosage strengths and frequencies limit the significance of our findings.

\section{Conclusions}

The advantages of the single acoustic wave treatment with its safety and high efficacy are demonstrated through objective 3-D volumetric assessments. Objectively, outcomes were demonstrable and impressive, with almost no downtime, discomfort and negligible side effects.

Unlike conventional medical procedures, this approach represents a safe and highly efficacious treatment for facial tightening without severe tissue damage, and is recommended as an alternative or adjunct treatment for facial tightening.

These advantages and clinical benefits of this approach would suggest that it would be easily adopted by socially active people and develop widespread acceptance by patients regardless of age, sex or skin type.

Overall, this single acoustic wave approach provides safe, long-lasting, and effective treatment of facial tightening.

\section{Conflicts of Interest}

The authors declare no conflicts of interest.

\section{References}

[1] Tanaka, Y. (2013) Objective Assessment of Skin Tightening Using Multisource, Phase-Controlled Radiofrequency in Asians. Journal of Cosmetics, Dermatological Sciences and Applications, 3, 110-116. https://doi.org/10.4236/jcdsa.2013.31016

[2] Tanaka, Y. (2015) Long-Term Three-Dimensional Volumetric Assessment of Skin Tightening Using a Sharply Tapered Non-Insulated Microneedle Radiofrequency Applicator with Novel Fractionated Pulse Mode in Asians. Lasers in Surgery and Medicine, 47, 626-633. https://doi.org/10.1002/1sm.22401

[3] Srisubat, A., Potisat, S., Lojanapiwat, B., Setthawong, V. and Laopaiboon, M. (2014) Extracorporeal Shock Wave Lithotripsy (ESWL) versus Percutaneous Nephrolithotomy (PCNL) or Retrograde Intrarenal Surgery (RIRS) for Kidney Stones. Cochrane Database of Systematic Reviews, 11, CD007044.

https://doi.org/10.1002/14651858.CD007044.pub3

[4] Torricelli, F.C., Danilovic, A., Vicentini, F.C., Marchini, G.S., Srougi, M. and Mazzucchi, E. (2015) Extracorporeal Shock Wave Lithotripsy in the Treatment of Renal 
and Ureteral Stones. Revista da Associação Médica Brasileira, 61, 65-71. https://doi.org/10.1590/1806-9282.61.01.065

[5] Speed, C. (2014) A Systematic Review of Shockwave Therapies in Soft Tissue Conditions: Focusing on the Evidence. British Journal of Sports Medicine, 48, 1538-1542. https://doi.org/10.1136/bjsports-2012-091961

[6] Antonic, V., Mittermayr, R., Schaden, W. and Stojadinovic, A. (2011) Evidence Supporting Extracorporeal Shock Wave Therapy for Acute and Chronic Soft Tissue Wounds. Wounds, 23, 204-215.

[7] Mani-Babu, S., Morrissey, D., Waugh, C., Screen, H. and Barton, C. (2015) The Effectiveness of Extracorporeal Shock Wave Therapy in Lower Limb Tendinopathy: A Systematic Review. The American Journal of Sports Medicine, 43, 752-761. https://doi.org/10.1177/0363546514531911

[8] Hexsel, D., Camozzato, F.O., Silva, A.F. and Siega, C. (2017) Acoustic Wave Therapy for Cellulite, Body Shaping and Fat Reduction. Journal of Cosmetic and Laser Therapy, 19, 165-173. https://doi.org/10.1080/14764172.2016.1269928

[9] Angehrn, F., Kuhn, C. and Voss, A. (2007) Can Cellulite Be Treated with Low Energy Extracorporeal Shock Wave Therapy? Clinical Interventions in Aging, 2, 623-630. https://doi.org/10.2147/CIA.S1721

[10] Kuhn, C., Angehrn, F., Sonnabend, O. and Voss, A. (2008) Impact of Extracorporeal Shock Waves on the Human Skin with Cellulite: A Case Study of an Unique Instance. Clinical Interventions in Aging, 3, 201-210.

https://doi.org/10.2147/CIA.S2334

[11] Christ, C., Brenke, R., Sattler, G., Siems, W., Novak, P. and Daser, A. (2008) Improvement in Skin Elasticity in the Treatment of Cellulite and Connective Tissue Weakness by Means of Extracorporeal Pulse Activation Therapy. Aesthetic Surgery Journal, 28, 538-544. https://doi.org/10.1016/j.asj.2008.07.011

[12] Adatto, M., Adatto-Neilson, R., Servant, J.J., Vester, J., Novak, P. and Krotz, A. (2010) Controlled, Randomized Study Evaluating the Effects of Treating Cellulite with AWT/EPAT. Journal of Cosmetic and Laser Therapy, 12, 176-182. https://doi.org/10.3109/14764172.2010.500392

[13] Nassar, A.H., Dorizas, A.S., Shafai, A. and Sadick, N.S. (2015) A Randomized, Controlled Clinical Study to Investigate the Safety and Efficacy of Acoustic Wave Therapy in Body Contouring. Dermatologic Surgery, 41, 366-370. https://doi.org/10.1097/DSS.0000000000000290

[14] Tanaka, Y., Tsunemi, Y., Kawashima, M., Tatewaki, N. and Nishida, H. (2013) Objective Assessment of Skin Tightening Using Water-Filtered Near-Infrared (1000-1800 nm) Device with a Contact Cooling and Freezer Stored Gel in Asians. Clinical, Cosmetic and Investigational Dermatology, 6, 167-176. https://doi.org/10.2147/CCID.S47299

[15] Tanaka, Y. (2019) Long-Term Objective Assessments of Skin Rejuvenation Using Solar Protection and Solar Repair Shown through Digital Facial Surface Analysis and Three-Dimensional Volumetric Assessment. Clinical, Cosmetic and Investigational Dermatology, 12, 553-561. https://doi.org/10.2147/CCID.S218176

[16] Chan, H.H., Yu, C.S., Shek, S., Yeung, C.K., Kono, T. and Wei, W.I. (2008) A Prospective, Split Face, Single-Blinded Study Looking at the Use of an Infrared Device with Contact Cooling in the Treatment of Skin Laxity in Asians. Lasers in Surgery and Medicine, 40, 146-152. https://doi.org/10.1002/1sm.20586 